\title{
Multi-Agent Systems for Resource Allocation and Scheduling in a Smart Grid
}

\author{
Arun Sukumaran Nair ${ }^{1} \cdot$ Tareq Hossen $^{1} \cdot$ Mitch Campion $^{1} \cdot$ Daisy Flora Selvaraj ${ }^{1}$. Neena Goveas ${ }^{1}$. Naima Kaabouch ${ }^{1}$. \\ Prakash Ranganathan ${ }^{1}$ (D)
}

Received: 3 March 2018 / Accepted: 16 October 2018 / Published online: 29 October 2018

(C) The Author(s) 2018

\begin{abstract}
With the increasing integration of Distributed Energy Resources (DER) in the power grid, a decentralized approach becomes essential for scheduling and allocation of resources in a smart grid. Economic Dispatch (ED) and Unit Commitment (UC) are the two major resource allocation problems that play critical role in the safe and stable operation of a grid system. The uncertainty associated with renewable energy sources have made the resource allocation problems even more challenging for grid operators. The future grid will have a higher generation mix of renewable energy sources and a large load of Electrical vehicles, with the possibility of bi-directional power flow. This complex smart grid system necessitates the development of a decentralized approach to resource allocation problem, which allows inter-node communication and decision making. Multi-agent systems (MAS) is a promising platform to decentralize the traditional centralized resource allocation aspects of smart grid. This paper presents a comprehensive literature review on the application of MAS to Economic Dispatch (ED) and Unit Commitment (UC) in smart grids.
\end{abstract}

Keywords Multi-agent systems $\cdot$ Smart grid $\cdot$ Resource allocation and scheduling $\cdot$ Economic dispatch $\cdot$ Unit commitment

\section{Introduction}

The smart grid framework incorporates distributed generation, advanced communication network, smart meters and sensors to make the grid more reliable, flexible, adaptive and efficient. This new power system paradigm necessitates the need to revisit some of the traditional power system operations to meet the challenges of next-generation transmission and distribution systems [1]. Along with the integration of renewable energy sources, the deregulation in the energy market has created competition for power generation companies. Generation companies have an obligation to meet the customer energy demands even during peak hours and system outages. There is a need to properly allocate the generation sources to maximize the profit considering renewable generation and customer demand [2].

Arun Sukumaran Nair

arun.sukumarannair@und.edu

1 Department of Electrical Engineering, University of North Dakota, Grand Forks, ND, USA
The presence of increased penetration of Renewable Energy Sources (RES) in the power system creates many technological challenges for the power companies owing to the need for improved system control to maintain the power quality to consumers [3]. The mix of conventional and RES must work in tandem to maintain the power generation at the required level. The process of committing the generators and allocating the required generation level has become a challenge to meet the increased demand, while using the generation from uncertain RES. These factors make the centralized control of a smart grid system complex and less efficient to process the diversity of data and controls [4]. The concept of Multi-Agent Systems (MAS) is put forward to solve this problem by using automated agent technology. The MAS converts a centralized control system into a distributed control model at a component level.

MAS is a collection of agents working together with each other to achieve an overall objective [5]. An agent can be defined as a computer system with the ability to take critical decisions based on the scenario to improve its objective $[6,7]$. These software agents in a smart grid environment sense, communicate, collaborate and act with each other. The agents can act autonomously or semi-autonomously, with local or 
global information [8]. MAS technology is finding wide range of applications in the power system domain such as optimal power flow $[9,10]$, power system restoration [11-17], electricity market operation [18-21], power system control [22-26] and protection [27-29]. The focus of this paper will be restricted to the application of MAS in the fundamental resource allocation aspects of the power grid namely, Economic Dispatch (ED) and Unit Commitment (UC).

ED is one of the most important challenges in the power systems and it deals with the allocation of power generation among committed generators in order to meet the demand while lowering the generation cost [30]. Consumer demand for clean energy and government regulation has motivated the integration of Distributed Energy Resources (DERs) like solar photovoltaic, wind power, and fuel cells into the modern power grid. This makes ED a highly complex optimization problem which needs to consider the various factors like generator capacity, ramp-rate, failure rate, emission, load profile and generation from DER. Unit Commitment (UC) in a smart grid system is a highly complex optimization problem that schedules the startup and shutdown of generators to meet the demand while satisfying system constraints [31, 32]. The committed generators are modeled in the ED for generator scheduling. The smart grid systems which have significant DER and the increased interest from consumers to install RES have necessitated the need for a decentralized approach to commit and schedule generators. The increased uncertainty from RES has made ED and UC more complicated due to the intermittent nature of these power sources.

For understanding the application of MAS in resolving resource allocation and scheduling problems of smart grid, the paper is organized in five sections. Section II describes the architecture of a MAS in a smart grid system, section III and IV present comprehensive review on the application of MAS in ED and UC respectively, followed by conclusion in section $\mathrm{V}$.

\section{MAS for Smart Grid}

An agent represents a computer system situated in an environment where it is capable of making decisions to achieve its design objectives. Moreover, an agent can be autonomous, social, reactive and proactive. Multi Agent Systems (MAS) are composed of agents interacting in a highly dynamic environment. These intelligent agents are being developed to have the functionalities on par with the human experts to act appropriately in the various scenarios that take place in a smart grid. The summary of various MAS architectures used for control of microgrid is summarized in Table 1 [33].
The centralized MAS architecture for micro grid control is discussed in the literature $[34,35]$. The framework of distributed and three level hierarchical MAS for a smart grid is explained in P. Lu et al. [36] and K. E. Nygard et al. [37] respectively.

Most of the MAS based optimization problems rely on the technique of consensus algorithm to reach the solution. The main idea behind the consensus problem is to make a set of agents agree up on a certain value (usually a global function) by using local information exchange among agents (local interaction). This concept is utilized in different fields such as economics (Agreement problem), communication (Gossip algorithms [38]), statistical mechanics [39] (Synchronization) and robotics (Flocking [40]). It offers several advantages over traditional centralized methods such as distributed computation, computational efficiency, independent of graph topology and robustness to failure [41, 42]. In a consensus algorithm model, each node in the system is considered as a dynamic agent with a value or state associated with it. The value of the agent represents the decision variable with which it can reach consensus with other agents in the system. Researchers have explored different census methods for a microgrid system such as in the work by G. Hug et al. [43] where a combination of consensus and innovation method was utilized. A novel framework to model a full automation of a distributed smart grid system is presented in the work by K. E. Nygard et al. [37]. The model is based on the concept of an Intelligent Autonomous Distributed Power System (IDAPS), a microgrid with sufficient resources and intelligence to function autonomously within a global grid. A three-layer hierarchical system model with agents in higher level supervising the agents in lower levels is proposed. The model accomplishes modularity, scalability, and a balance between global and local decisions of agents. Distributed MAS based control offers several advantages such as autonomy, fault-tolerance low latency, efficiency and much more. It is a way of physically breaking complex control problems into smaller control problems, and then solving them closer to the control operation itself.

Development of Smart Grids will involve dealing with a big amount of data collected in a distributed manner. This data is communicated among equipment and devices to support decision-making process. Certainly, handling the amounts of data to be acquired and processed in such distributed systems to extract useful information bring its own challenges. Computational intelligence techniques are used to extract knowledge and overcome some of the challenges. In "intelligent" systems, data is pre-processed, processed, and then information is extracted for decision-making. Given the distributed nature of Smart Grids (SG), new advancements in distributed intelligence techniques spawned the MAS technique development. The following are some advantages and reasons to explore distributed MAS architectures over centralized architecture: 
Table 1 Different MAS architectures for microgrid control [33]

\begin{tabular}{|c|c|c|c|}
\hline MAS architecture & Type of agent & Role & Features \\
\hline \multirow[t]{2}{*}{ Centralized } & Cognitive Agent & $\begin{array}{l}\text { Higher level of intelligence/ } \\
\text { communication capabilities }\end{array}$ & $\begin{array}{l}+ \text { collects information } \\
\text { at a single point }\end{array}$ \\
\hline & Reactive Agent & Fast Response & $\begin{array}{l}\text { + capable of making global decision } \\
+ \text { flexibility and openness in } \\
\text { the operation of smart grid } \\
\text { - suffers from computational burden } \\
\text { in case of large number of agents } \\
\text { - single point of failure affects the } \\
\text { entire system }\end{array}$ \\
\hline \multirow[t]{2}{*}{ Two-level hierarchical } & High level agent & $\begin{array}{l}\text { Infrastructure management, } \\
\text { low level scheduling }\end{array}$ & \multirow{2}{*}{$\begin{array}{l}\text { + distinct levels of decision making } \\
\text { - failure of higher level agents results } \\
\text { in critical conditions of the lower level agents }\end{array}$} \\
\hline & Low level agent & $\begin{array}{l}\text { Accept schedule from High } \\
\text { level agent, asset management }\end{array}$ & \\
\hline \multirow[t]{3}{*}{ Three-level hierarchical } & High level agent & $\begin{array}{l}\text { Critical decisions, data and } \\
\text { policy management }\end{array}$ & \multirow{3}{*}{$\begin{array}{l}\text { + good scalability through delineation } \\
\text { of roles to agents } \\
\text { - failure of higher level agents results in } \\
\text { critical conditions of the lower level agents }\end{array}$} \\
\hline & Middle level agent & $\begin{array}{l}\text { Fault location, switching of grid } \\
\text { connected/islanded mode }\end{array}$ & \\
\hline & Low level agent & Sensor management & \\
\hline Distributed & Local Agent & $\begin{array}{l}\text { Local information discovery/ } \\
\text { communication }\end{array}$ & $\begin{array}{l}\text { + robust system with agents being capable } \\
\text { of reorganizing and coping up with the } \\
\text { loss of other agents }\end{array}$ \\
\hline
\end{tabular}

\section{Advantages of Distributed MAS over centralized control:}

1. The SG components are often distributed and the energy management system is tightly associated with the communications between stakeholders and entities (agents) to exchange information, so MAS is an appropriate platform to develop distributed management functions.

2. SG is a holistic system and the failure of some part of it (e.g., the breakdown of a transmission line or cut down of a substation, transformer) should not affect the whole activities and operations, and hence fault tolerance can be easily attained in distributed architecture over centralized architecture.

3. SG should demonstrate the plug-and-play concept for integrating energy storage, loads, and sources at the building level with the external utility grid. Plug and play adaptability is widely proven by MAS. The nature of MAS enables it to scale up by adding other agents or by dispersing them in new environment with new resources and capacities. Hence, Distributed MAS building modules are highly scalable, and modular.

4. As SG will be composed of an aggregate of Micro grid, and hence the control can be delegated to micro grids. With futuristic smart grids being a simple collection of residential microgrids, each microgrid can exhibit distributed control.

5. One of the goals of the SG is to develop grid modernization technologies, tools, and techniques for Demand-
Response (DR) with the ability to dynamically optimize grid operations, resources and consumer participation. To do so, it is important to understand demand participation of consumers. As number of consumers are growing, it is essential to do the demand response analytics in a distributed fashion.

Due to the inherent advantages of distributed MAS architecture, it is well suited to resolve the complex ED problem of smart grid. A general framework for the implementation of MAS in smart grid (SG) system is shown in Fig. 1 [44]. SG system is an integration of the physical grid with the communication layer where the agents act as an interface. The communication layer is a strongly connected network with varying and configurable topologies. Each agent can be categorized into three units; namely Device Unit (DU), Decision Making Unit (DMU) and Communication Unit (CU). DUs can be considered as physical power system buses with components such as Synchronous Generators (SGR), Renewable Generators (RG), flexible load and rigid load. DMUs perform the local computing for the agents and CUs are the communication nodes, which transmit and receive information [45].

The internal structure of an agent model is shown in Fig. 2. An agent model consists of three units; Communication Unit (CU), Decision Making Unit (DMU) and Device Unit (DU). CUs are generally signal receivers/transmitters used to exchange information with neighbors. The calculator, sensors and controllers are part of DMU, which are responsible for the local computing in an agent. DMU is the brain of an agent node and capable of generating control instructions for the DU 
Fig. 1 Framework for distributed multi-agent system for smart grid [44]

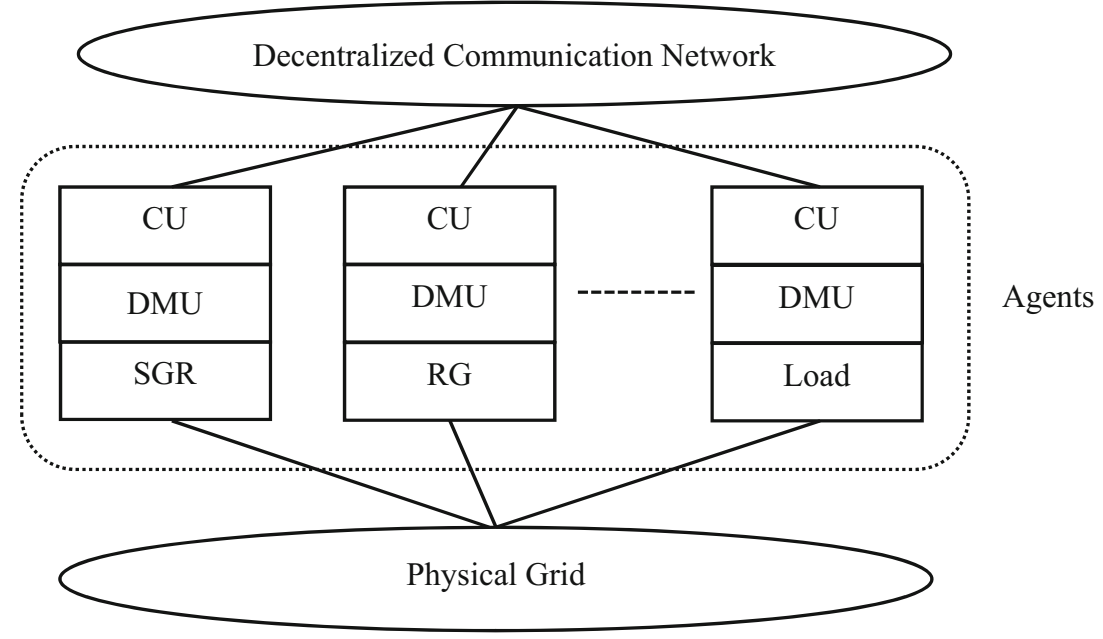

as well as responsible for communicating the information to the CU. DUs represent the traditional buses in a network which consists of different elements such as synchronous generators, Renewable Generators (RG), battery storage systems (BESSs), flexible and rigid loads. DU performs the control suggestions from DMU and also sends the feedback to the DMU.

There are number of simulation and open source tools available for modeling MAS platforms [46]. The most common ones are ZEUS, AgentBuilder, JADE, and MADKit. Features of these MAS modeling tools are listed in Table 2.

\section{Economic Dispatch}

Economic Dispatch (ED) is one of the fundamental problems in the power system domain. It is basically an optimization problem with the objective of reducing cost while maintaining the generation-load balance. ED schedules the committed generators in the system to meet the demand. ED needs to conform to several other constraints for a safe and secure operation of the grid. The integration of uncertain renewable energy sources to the grid has made ED and power quality analysis more important and also more complicated [52, 53].

Distributed algorithms are becoming popular for intelligent decision-making and control and these algorithms appear to be promising in the context of smart grid. These algorithms are robust, immune to topological variations and can support the "plug-and-play" feature of the future grid. However, it is more challenging to include the operational constraints in such a distributed formulation. Many researchers have proposed a consensus-based approach for ED without losses and lower and upper power boundaries. A consensus algorithm is widely used in solving the ED problem in smart grid. It is a method used to achieve agreement on a single data value among distributed systems. This algorithm is designed to achieve reliability in a network involving multiple unreliable nodes. [36, 54-56].
Fig. 2 Internal structure of an agent [44]

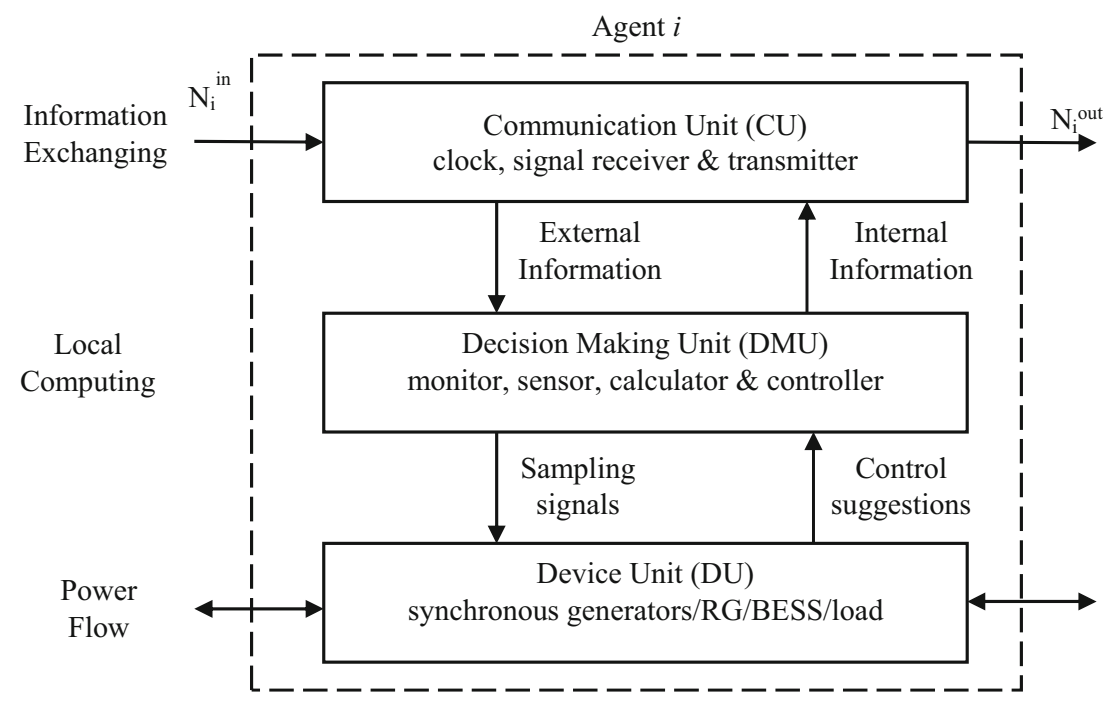


Table 2 MAS modeling tools

\begin{tabular}{|c|c|}
\hline MAS tools & Description \\
\hline ZEUS & $\begin{array}{l}\text { ZEUS [47] is a multi-agent platform developed by the research program of } \\
\text { British Telecom intelligent system research laboratory. ZEUS allows the } \\
\text { design of multi-agent distributed systems. This platform, developed in Java, } \\
\text { automatically generates Java code from the agents specified graphically. }\end{array}$ \\
\hline Agent Builder & $\begin{array}{l}\text { Developed by Reticular Systems Inc., AgentBuilder [47], it is based on BDI } \\
\text { (Believe - Desire - Intention) models Agent [48] and AGENT-O language [49]. } \\
\text { It is remarkable for the quality of its software and a good academic model. } \\
\text { AgentBuilder is a commercial design software for "intelligent" agents i.e., } \\
\text { cognitive and collaborative agents. AgentBuilder consists of two main components: } \\
\text { the toolkit and runtime system. }\end{array}$ \\
\hline JADE & $\begin{array}{l}\text { JADE [50] is a multi-agent (multi-host) platform developed by Bellifemine. } \\
\text { F., Poggy. A., Rimassa. G. and P. Turci by Telecom Italia Lab "Tilab formerly CSELT" } \\
\text { in 1999. This platform aims to simplify the construction of interoperable MAS, achieve } \\
\text { compliant applications with the standard FIP A97 (Foundation for Intelligent Physical Agents) } \\
\text { to facilitate the communication of JADE agents with non-JADE agents, and optimize the } \\
\text { performance of a distributed system agent. JADE includes all accredited component that } \\
\text { manages the platform: Agent Communication Channel (ACC), Agent Management System } \\
\text { (AMS), and Director Facilitator (DF). }\end{array}$ \\
\hline MADKit & $\begin{array}{l}\text { MADKit [51] is a platform for MAS developed by Olivier Gutknecht and Jacques } \\
\text { Ferber in Laboratory of Computer Science and Robotics and Microelectronics of } \\
\text { Montpellier. MADKit was motivated by the need for a more flexible platform possible, } \\
\text { and able to adapt to different agent models and application areas. MADKit is a modular } \\
\text { multi-agent platform and scalable, written in the Java language. It allows the creation of MAS } \\
\text { based on the relational model Aalaadin or AGR (Agent / Group / Role): agents are located in } \\
\text { groups and play roles. MADKit takes advantage of object-oriented programming: MADKit } \\
\text { features are contained in the MADKit kernel. }\end{array}$ \\
\hline
\end{tabular}

In a conventional centralized method (e.g. Lagrange multiplier method), at the optimal point, all the generators will have the same incremental cost. An appropriate consensus algorithm can guarantee a similar result by having all the consensus variables to converge to a common value asymptotically. Based on this concept, Z. Zhang and M. Chow [57] introduced an Incremental Consensus Algorithm (ICC) to decentralize the ED problem by choosing incremental cost (IC) as a consensus variable. The model consists of a local controller (generation unit) which will update its consensus variable depending up on the neighbor's values. The proposed approach requires a leader node, which will decide whether to increase or decrease the IC based on the demand constraints. The authors tested the approach on a 3 unit and 5 unit system to test the validity and convergence of the proposed approach. They showed a successful implementation of consensus algorithm in ED but lacked a fully distributed model since it needed a leader node to control the agents. A more detailed description about ICC is provided in [58].

The previous paper utilized ICC algorithm to implement ED in a distributed fashion but relied on a leader-follower consensus algorithm. A leader node needs to be selected, which will gather the local power mismatch from the follower nodes to calculate the total power mismatch. The follower nodes need to report their power mismatch to their leader. $\mathrm{Z}$. Zhang et al. [59] introduced a two-level consensus approach to acquire system power mismatch. This new approach will eliminate the need for a single leader node to do all the calculation. An average consensus will run at the lower level of the two-level method and ICC will be employed at the second level to process the mismatch information. This is an improved version from the method proposed in [57]. It is more distributed and does not require a fixed communication network.

A decentralized approach to ED in a microgrid with Distributed Generators (DG) was explored by N. Cai et al. [7] using a MAS. Here, each DG was assumed to have an agent which could receive local information and communicate only with its nearest neighbors. Agents compete with others to obtain a local solution, thereby obtaining a global optimum. The authors used the concept of consensus among agents to obtain the optimum solution for the ED. The authors validated the approach on five and fifty agent systems but did not compare the results with a centralized approach.

A consensus control based approach to solve the ED problem in a smart grid was developed by S. Yang et al. [60]. The approach solves the ED in a distributed fashion with the generators acting collectively to receive the mismatch between demand and power generation information, which is the feedback for the agents. The total mismatch is generated in a collective fashion from the estimate of local mismatch by the agents, which removes the need for a leader agent to collect 
the global information. The incremental cost of the generators is chosen as the consensus variable and incremental cost criterion was used to obtain the optimal dispatch. The method was found to have the same precision as the Lambda-Iteration approach, a centralized method, with less communication overhead.

A distributed ED model considering line loss was developed by G. Benetti et al. [61]. The nodes in the model run two consensus methods in parallel; one to find the Lagrangian variable and a second one to find the power mismatch. The first method is a first-order consensus algorithm which uses a proportional controller to bring the power mismatch to zero and to satisfy the generation-demand equality constraint. The second consensus method uses the work allocation concept to find the power mismatch. The authors assert that the proposed method can satisfy generation constraint and can handle line loss in the system. The comparison between the distributed approach and the centralized approach to verify its convergence speed and accuracy was not attempted by the authors.

A. Cherukuri et al. [62] explored the concept of distributed consensus-based approach to model an ED which can handle changing load conditions and can remain stable under intermittent power sources. The proposed model employs two dynamical systems namely, dynamic average consensus and Laplacian non-smooth gradient. The mismatch between generation and load is estimated in a distributed fashion by the consensus method and the Laplacian non-smooth gradient dynamically allocates the generation. The approach can reach optimum solution from any initial power allocation and does not require a feasible allocation as the initial value. The authors verified the effectiveness of the method to handle dynamic loads and intermittent power sources.

K. Luo et al. [63] developed a MAS based distributed ED model for an electrical grid system with RES, which can be deployed for real-time applications. The proposed approach is a two-step process, with the first step calculating the initial generation values using adjacency average allocation algorithm and the second stage performs the ED in a distributed manner using local replicator dynamics. The first stage handles the equality constraints in the model while the second stage conforms to the inequality constraints. They validated the effectiveness of the proposed method but did not compare the performance of the method with similar approaches.

A distributed ED model for an islanded microgrid system was developed by P.P. Vergara et al. in [64]. The model considered both active and reactive power in the optimization model. The primal-dual constrained optimization method was used to solve the problem in a distributed fashion, in which two consensus methods are executed in parallel to obtain the dual values or incremental costs. The authors validated the performance of the proposed method by comparing to a classical Lambda method and also the capability of the method for fault tolerance.
F. Guo et al. [65] explored the potential of a distributed ED model for a smart grid system with random wind power. The proposed model works on the projected gradient and Finitetime Average Consensus Algorithm (FACA) and supports the plug-and-play feature of new generation smart grids. The random wind power generation is modeled using the deterministic method with overestimation and underestimation cost variables. The agents can choose arbitrary initial values and are not required to share gradient or incremental cost information with the neighbors. The authors validated the effectiveness and performance of the proposed method on IEEE test systems.

A consensus-based distributed ED taking into account generator dynamics was studied by J. Cao et al. in [66]. The authors used comprehensive generator constraints to improve the consensus algorithm and analyzed the effect of different communication topologies on the speed of the consensus algorithm. The model relies on local power mismatch data from the agents rather than a leader node to collect global information. The authors asserted the superiority of the proposed method by comparing with Lambda iteration and PSO methods. The generator dynamics was found having a significant effect on the speed of the consensus method while the effect of communication topology was not significant.

A distributed consensus-based approach to solve ED in a microgrid was developed by Z. Yang et al. [67]. They used a novel concept of virtual incremental cost as the consensus variable which does not require the nodes to share power output or generator parameters. The algorithm has the advantage of not depending on the local power mismatch to reach the optimum and maintaining the supply-demand balance even during transients. They reported reduction in communicational burden between nodes and improved reliability of the algorithm.

Y. Li et al. [68] developed a distributed ED model for a combined heat and power system. The MAS based framework utilized two consensus protocols, one optimizes the electrical incremental cost function while the other gets a common value for the heat incremental cost. The heat and power coupling in the objective function and constraints are managed by these two consensus variables. It works in a completely distributed fashion without the need for a leader agent with the global information. They report the effectiveness of the proposed ED model by comparing to a centralized approach using Lagrangian relaxation method.

Z. Yang et al. [69] proposed a distributed consensus-based model for the ED in a smart grid system which maintains the supply-demand balance even during the transient process. The method has the advantage of not relying on the supply-demand mismatch and hence can be used online. The proposed method does not require a leader node with the complete information of power demand in the grid system. It uses the maximum incremental cost of the neighboring generators and developed a 
method to increase or decrease the incremental cost of a saturated generator to maintain the supply-demand balance during iterations. H. Xing et al. [70] utilized an average consensus based bisection approach to perform distributed ED. The method has the advantage of not relying on prior information or a leader node to perform the optimization.

G. Binetti et al. [71] developed a distributed model to solve non-convex ED problems. The non-convexity comes from the valve-point effect, prohibited operating zones, multiple fuel option and transmission losses but makes the model more realistic for real-time operations. The proposed model is fully decentralized and does not require a leader node with the global information. The method has the added advantage of being deterministic while heuristic methods do not guarantee the uniqueness of the solution from a single run. A combination of auction mechanism and marketbased MAS was used to design the distributed ED and the authors tested the validity of the method on standard test systems. G. Binetti et al. [72] also proposed a distributed ED model which considered transmission losses in the system using a combination of two consensus algorithms running in parallel. The model utilized a first order consensus protocol to calculate the local power mismatch to satisfy the demand constraint and a second consensus algorithm to calculate the system power mismatch.

A transition of the MAS based distributed ED from laboratory set up to industrial model is studied by G. Zhabelova et al. [73]. An incremental cost consensus approach model based on the industrial standard IEC 61499 is used to solve ED in a smart grid environment. IEC 61499 is a promising industrial standard used as an architecture for the development of distributed systems in control and automation. The agentbased system modeled after the IEC 61499 standard will be suited for industry application and can be executable on the target platforms. The authors tested the proposed model on a 5-node system with industrial controllers.

A combination of MAS and Particle Swarm Optimization (PSO) called MAPSO (Multi-Agent Particle Swarm Optimization) was proposed by $\mathrm{C}$. Wu et al. [74] and was applied to the ED problem. The proposed method overcomes the shortcomings of PSO, the fast convergence to the local optima, and achieves high convergence speed and precision. The agents are modeled to have the ability of self-learning to improve the problem-solving ability. The authors verified the effectiveness of the proposed method on IEEE test buses and the method was found to be faster than evolutionary algorithms. A hybrid of MAS with PSO, deterministic search and bee decision-making process called HMAPSO (Hybrid Multi-Agent based Particle Swarm Optimization) was proposed by R. Kumar et al. [75]. The HMAPSO method was applied to an ED model with valve-point effect and was observed to be more robust and accurate than other PSO methods.
A dynamic agent-based approach to model a decentralized ED was developed by V. Loia et al. [76]. ED was solved using self-organizing dynamic agents equipped with distributed consensus method. C. Zhao et al. [77] explored the effect of cyber-attacks on a consensus-based ED model. The authors tested the performance of the algorithm for false data injection into the broadcast information, offline and online ED models, and bounded and unbounded generation cases.

The increased amount of communication between nodes in a smart grid system can lead to communication bottlenecks which can cause convergence issues in consensus-based ED models. C. Li et al. [78] developed an event triggered consensus-based ED model to reduce the communication overhead in a smart grid system. The authors reformulated the ED model using $\theta$-logarithmic barrier to conduct the information exchange in a distributed fashion. The reformulated ED model is solved in a two-stage process; the initial values for the agents are generated using connected dominating set based distribution algorithm as the first stage and in the next stage a consensus-based optimization is applied to the system. The authors stated that asynchronous communication-based event triggered ED model can significantly reduce the communication exchange in a smart grid system, but the event triggered mechanisms can have a negative impact on the convergence rate. A fast gradient-based method is used to accelerate the convergence rate in the optimization model.

Most of the papers discussed above assume a perfect communication between agents without any information loss, but in a realistic smart grid environment can have packet loss and communication failures. Y. Zhang et al. [79] proposed a distributed ED model which remains robust under information loss among agents. A combination of two algorithms running in parallel, Robust distributed system Incremental Cost Estimation (RICE) algorithm, was introduced by authors to handle the issue. The model contains a Gossip algorithm to find the power mismatch estimation and consensus algorithm for the incremental cost estimation. They report that their method outperforms the consensus method to packet loss and delivered good results even with a 5\% information loss in the network. Another study on distributed ED under communication uncertainties is by G. Wen et al. [80]. The proposed approach utilized a robust consensus model to counter the communication uncertainties. A study of consensus-based ED model under dynamic communication network is evaluated by M. Hamdi et al. in [81]. T. Yang et al. [82] explored a distributed ED model for a system with potentially timevarying topologies and network delays. The authors proposed a gradient push-sum based method to handle the network challenges.

The application of distributed ED using consensus theory in a microgrid is by proposed R. Wang et al. [83]. The proposed method is a fully distributed approach without a leader or a virtual control node. The incremental cost of 
each bus in the system is taken as the consensus variable. The authors validated the performance and convergence of the distributed ED in a microgrid model. A similar approach for distributed ED in an islanded microgrid is proposed by Z. Tang et al. [84] by using IC as the consensus variable. A distributed power dispatch model for a multi-microgrid scenario is reported by $\mathrm{X}$. He et al. [85] utilizing a primal-dual consensus algorithm. They evaluated the performance of the proposed algorithm on an IEEE 30, 57 and 300 bus systems. A study on distributed control architecture for a hybrid AC/DC microgrid is performed by P. Lin et al. in [86]. In most of the above cited papers for ED, an evaluation of generation cost is not performed for the different approaches and moreover the problem of ED is not solved in a more realistic manner with non-negligible losses. Limited work has been done on the implementation cost of these approaches in a smart grid system. A summary of the above cited papers and their features are listed in Table 3.

\section{Unit Commitment}

Unit Commitment is the process of determining the schedule of generating units within a power system. The optimized schedule is generated subject to device and operating constraints of the system with the objective of minimizing the cost for utilities [92]. The ED optimization is usually performed on the committed generators from the UC step. Various approaches were used to find the optimal schedule from the UC problem ranging from highly complex and theoretical methods to simple rule of thumb methods [93-97]. The scope of the UC problem depends on the generation mix, operating and security constraints set by the utility. The focus of this review is on the decentralized approaches which utilized MAS to solve UC problem.

Authors in [87] developed a centralized approach to solve UC in a smart grid system using a MAS based architecture. The agents communicate information to neighboring agents, but the UC happens in a centralized controller. Figure 3 shows the different agents in a centralized UC. The proposed method helps to reduce the communication overhead but has the demerits of a centralized controller such as a single point of failure, increased computation time with complexity, unavailability of plug-and-play functionality etc.

A distributed UC model based on MAS was developed by T. Nagata et al. [31, 98]. The proposed model consists of three types of agents namely Generator Agents (GA), mobile agents (DA, UA) and Facilitator Agent (FA). The FA contains the objective function. The system level constraints are satisfied by the interaction between GA and mobile agents and the GA handles the local constraints. The two mobile agents are provided to improve the communication in the system. The decrease mobile agent (DA) are intended to reduce the generated output and increase mobile agents (UA) initiates an increase in generated output. In the proposed approach, mobile agents travel throughout the system and negotiates with the generator agents, depending up on the operating conditions. The performance comparison of the method with dynamic programming yielded similar results but it is not a fully decentralized method since it has a leader and mobile nodes as an interface between generator agents. Figure 4 shows the architecture of the proposed method.

An improved version of the method proposed in [31] was presented by J. Yu et al. in [88]. The proposed MAS agents are more intelligent and have the capability to solve complex optimization problems. The profit maximization objective is obtained using three types of agents, namely central agent, mobile agent and generator agent. The central operator in the system is the central agent which commands the mobile agents to achieve the objective function. The mobile agents travel to each generator to negotiate and reach a satisfactory result. The model is not fully decentralized as there is an agent acting as a central controller. The authors validated the proposed model against a hybrid Lagrange Relaxation - Evolutio nary Programming (LR - EP) method.

A MAS based UC model for a smart grid system considering RES uncertainty was developed by X. Zhang et al. [89]. The model considered the uncertainty associated with wind, solar and load along with the charging and discharging of PHEVs (plug-in hybrid electric vehicles). The hierarchical system consisted of management agents, work agents, cooperative co-evolution agent and object cooperative agent. The work agents used adaptive GA to solve the optimization.

A MAS based approach to solve the profit based UC problem was explored by J. Yu et al. [99]. Rule-based, and dynamic programming methods were used to solve the profit based UC. D. Sharma et al. [91] introduced an improved version for the profit based UC. The ISO agents in the proposed method used a rule-based intelligence to work in conjunction with generator agents to maximize the objective function. The functionality of generator agents is limited to maximize their profit for a given demand and reserve using real - parametric Genetic Algorithm and to share the information with the ISO agents. The maximum profit generating agents are committed to the system by ISO agents while satisfying the up/down time constraints. The authors reported the performance of the proposed method with several hybrid methods.

T. Logenthiran et al. [2] utilized MAS concept to develop a resource scheduling model for an islanded power grid with integrated microgrids and DER. The proposed methodology has three stages; microgrid scheduling to 


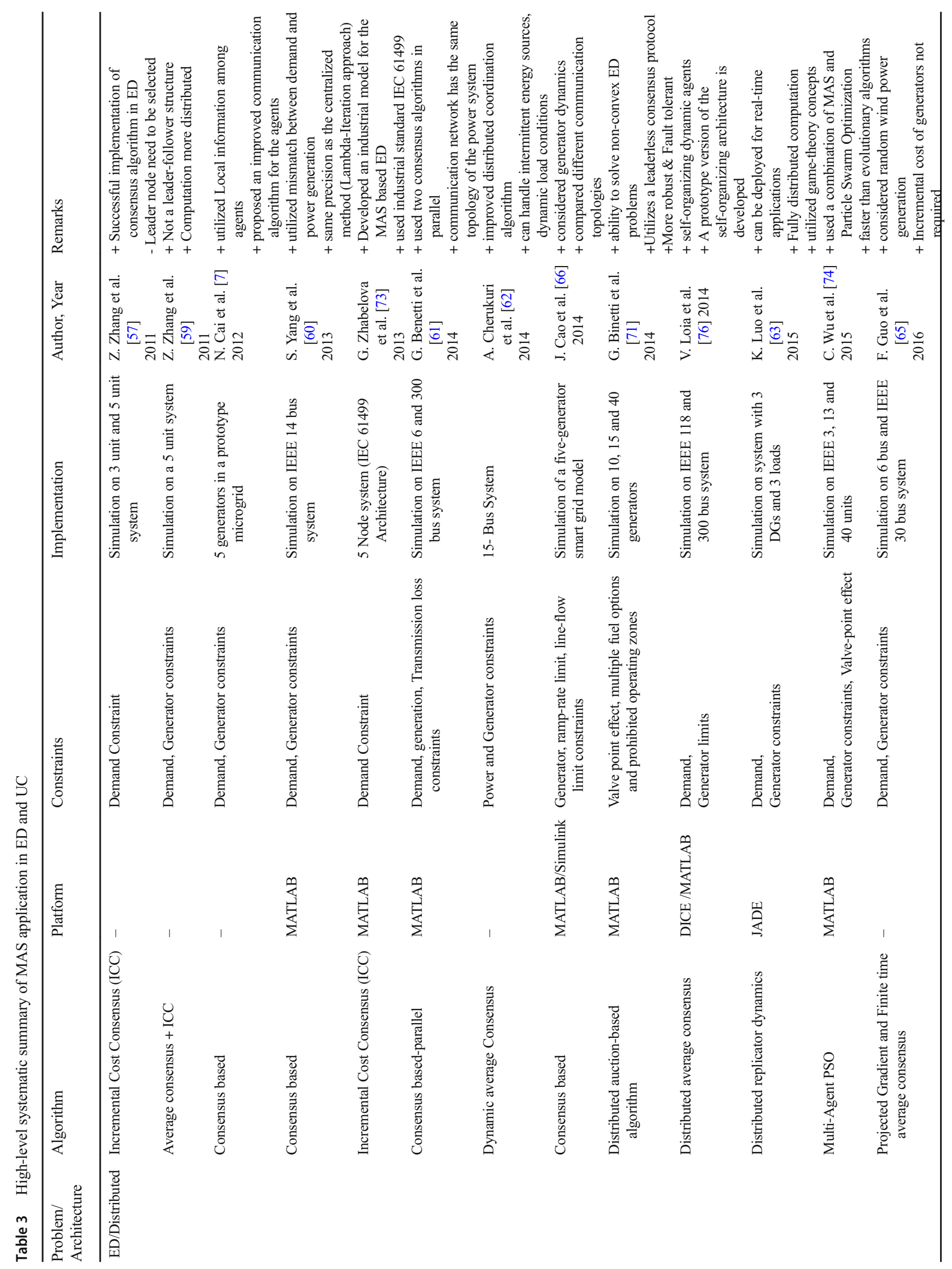




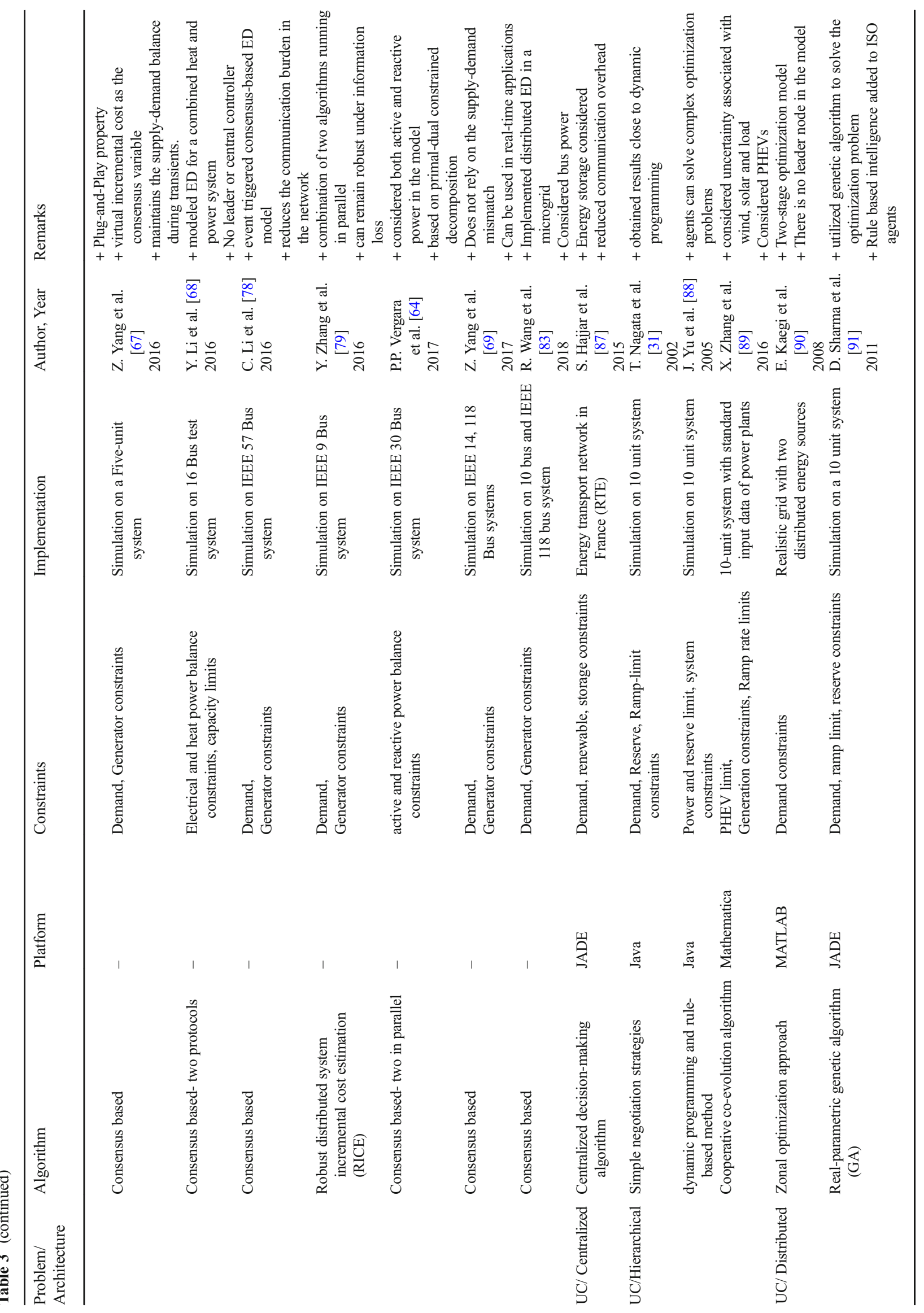


Fig. 3 Smart grid communicating agents in a centralized UC [87]

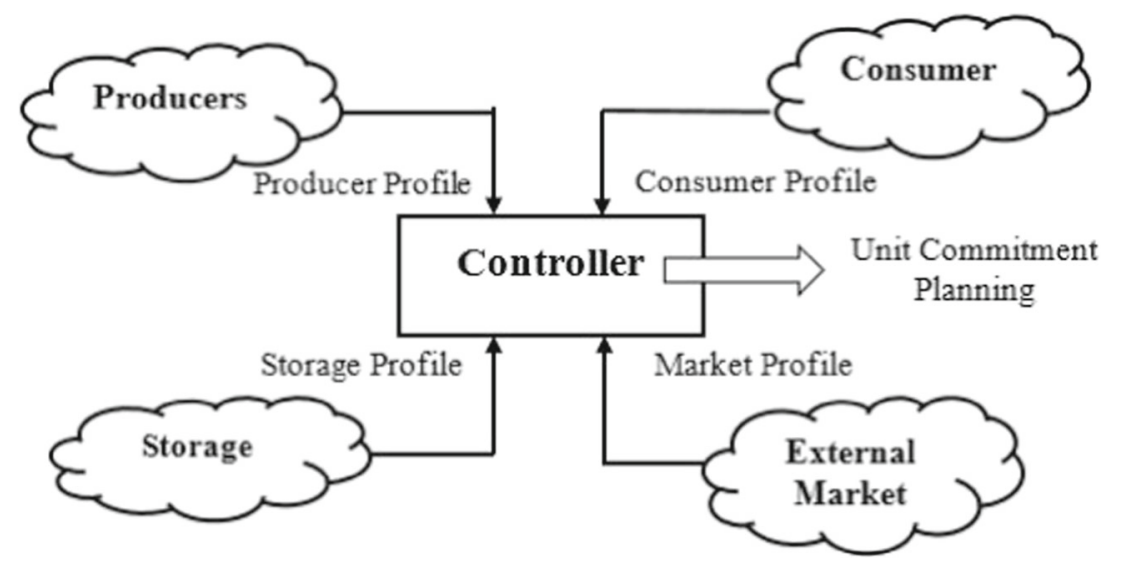

satisfy its internal demand as the first step, the second stage being contacting the network to analyze the possibility of exporting power, and the final step is to schedule the whole microgrid considering both internal demand and the power transfer from the second stage. The authors used the JADE platform to simulate the MAS system and used Lagrangian Relaxation with Genetic Algorithm to schedule the microgrid resources internally. They report the robustness and scalability of the method by testing it in a PoolCo energy market.

E. Kaegi et al. [90] proposed a decentralized approach to solve the UC problem using the MAS concept. The methodology was based on zonal approach consisting of generator agents, load agents and zone agents. The generator agents (GA) and load agents (LA) handle the local profit maximization within a zone while the zone agent handles the interaction with other zones. The zone agents have no financial objectives but only acts as a service agent for the entities within its zone. The optimization is done in two stages. The intra zone level is the competition between agents within a zone to reach the profit maximization. In the next stage, the optimization happens during the interaction between zones. The paper only focused on the intra zonal activities, but the inter-zonal activities also play a significant role in profit maximization. Figure 5 represents the zonal approach used by the authors. A summary of the work done by different researchers on ED and UC problem which utilized MAS concepts are summarized in the Table 3.

\section{Conclusion}

This paper presented a literature review on the application of MAS for ED and UC in a smart grid. The integration of DERs into a grid requires a decentralized control strategy to incorporate these resources and to maintain the grid resiliency. The multi-agent technology is a promising and scalable platform to implement distributed resource scheduling and allocation using various computational techniques.

Fig. 4 MAS based UC model

[31]

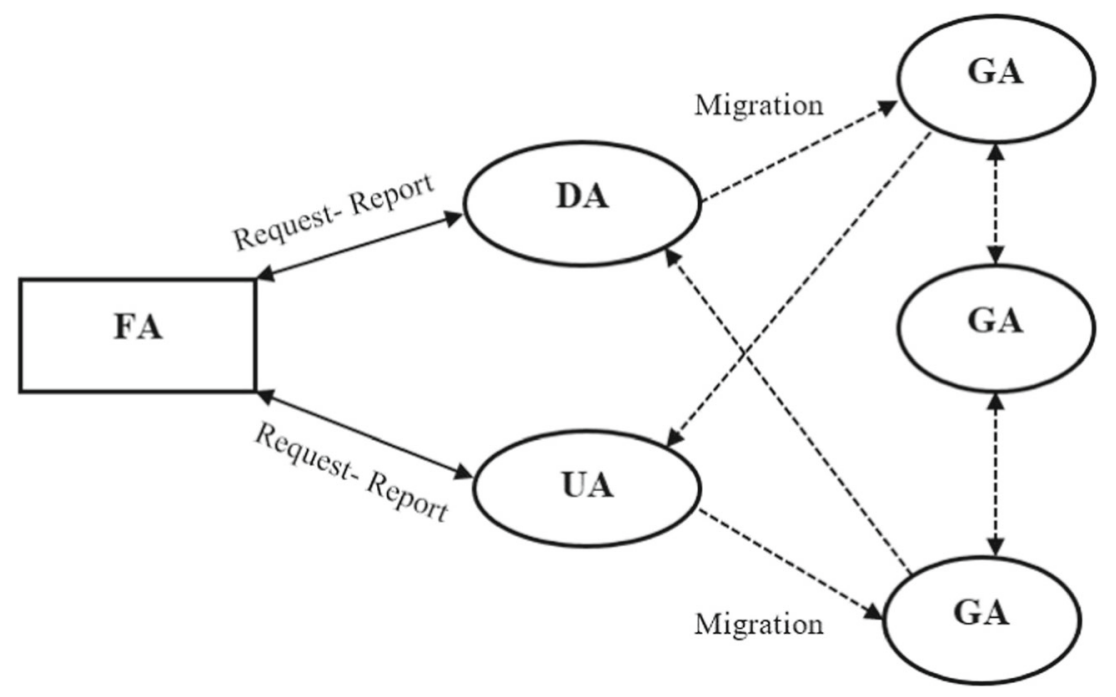


Fig. 5 Agent hierarchy in the UC Model [90]

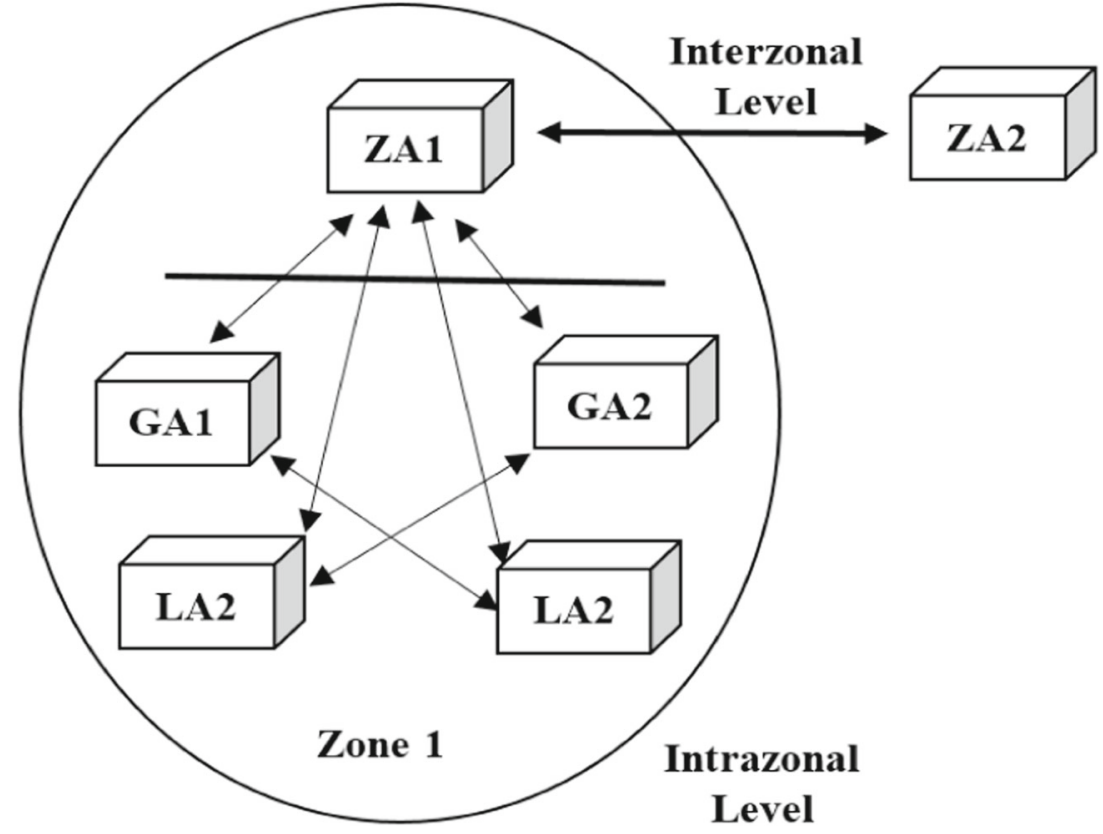

Though there are many centralized algorithms being used to solve the ED problem, a small change in the smart grid may lead to redesign of these centralized approaches. Thus, there is a need for a distributed ED approach which can enjoy the benefit of robustness, scalability and less information requirement. Different distributed algorithms for solving ED problem have been proposed by many researchers in the literature. Of all these distributed approaches, consensus-based algorithm has evolved as the promising computing method for solving ED. The consensus-based ED algorithm can make the analysis tractable by simplifying the system into linear for the iteration process. Most of the consensus-based algorithms available in the literature are useful in solving only convex ED problem without transmission losses. On the other hand, an auction-based algorithm has been proposed to solve nonconvex ED problem. However, most of the investigations reported in the literature are limited to implementation in the simulation environment without addressing the challenges of different scenarios of a smart grid in real time. Hence, these approaches have to be established in real time which would be helpful in solving ED problem in a smart grid.

Researchers have explored the application of MAS in centralized, hierarchical and distributed models for an UC problem. Most recent works focused on a distributed model utilizing concepts from the negotiation strategies and genetic algorithm. The distributed UC models used a zonal approach consisting of controller/zonal agents facilitating the communication among agents and these models are not completely distributed. There is a need for a completely distributed UC model which considers the increased DER penetration into the future grid. Most of the reviewed articles focused on the implementation and convergence ability of the proposed methods, more work needs to be done on evaluating these methods for their speed and cost savings in a real -time environment.

We believe that this paper can act as a resource for researchers in academia and analysts in utilities to understand the background on MAS's application for smart grid management and control.

Acknowledgments The authors acknowledge the support of the National Science Foundation (NSF) award \#1537565 for this work.

Open Access This article is distributed under the terms of the Creative Commons Attribution 4.0 International License (http:// creativecommons.org/licenses/by/4.0/), which permits unrestricted use, distribution, and reproduction in any medium, provided you give appropriate credit to the original author(s) and the source, provide a link to the Creative Commons license, and indicate if changes were made.

\section{References}

1. Xu Y, Zhang W, Wenxin L (2015) Distributed Dynamic Programming-Based Approach for Economic Dispatch in Smart Grids. IEEE Trans Ind Informatics 11:166-175. https://doi.org/10. 1109/TII.2014.2378691

2. Logenthiran T, Srinivasan D, Khambadkone AM (2011) Multiagent system for energy resource scheduling of integrated microgrids in a distributed system. Electr Power Syst Res 81: 138-148. https://doi.org/10.1016/j.epsr.2010.07.019

3. Arriagada E, Lopez E, Lopez M et al (2015) A probabilistic economic dispatch model and methodology considering renewable 
energy, demand and generator uncertainties. Electr Power Syst Res 121:325-332. https://doi.org/10.1016/j.epsr.2014.11.018

4. Nair AS, Ranganathan P, Kaabouch N (2017) A constrained topological decomposition method for the next-generation smart grid. In: 2017 Int. Conf. Electr. Comput. Commun. Technol. (IEEE ICECCT 2017). Coimbatore, pp 1-6. https://doi.org/10.1109/ ICECCT.2017.8118053

5. Sujil A, Verma J, Kumar R (2016) Multi agent system: concepts, platforms and applications in power systems. Artif Intell Rev 1-30. https://doi.org/10.1007/s10462-016-9520-8

6. Wooldridge M (2009) An introduction to multiagent systems. https://www.cs.ox.ac.uk/people/michael.wooldridge/pubs/imas/ IMAS2e.html. Accessed 5 Jan 2018

7. Cai N, Nga NTT, Mitra J (2012) Economic dispatch in microgrids using multi-agent system. 2012 North Am Power Symp NAPS 2012. https://doi.org/10.1109/NAPS.2012.6336435

8. Bou Ghosn S, Ranganathan P, Salem S, et al (2010) Agent-oriented designs for a self healing smart grid. 2010 First IEEE Int Conf Smart Grid Commun. https://doi.org/10.1109/SMARTGRID. 2010.5622085

9. Leeton U, Kulworawanichpong T (2012) Multi-Agent Based Optimal Power Flow Solution. In: 2012 Asia-Pacific Power Energy Eng. Conf. pp 1-4. https://doi.org/10.1109/APPEEC. 2012.6307223

10. Mohammadi J, Hug G, Kar S (2016) Agent-based distributed security constrained optimal power flow. IEEE Trans Smart Grid 9: 1118-1130. https://doi.org/10.1109/TSG.2016.2577684

11. Ren F, Zhang M, Soetanto D, Su X (2012) Conceptual design of a multi-agent system for interconnected power systems restoration. IEEE Trans Power Syst 27:732-740. https://doi.org/10.1109/ TPWRS.2011.2177866

12. Nagata T, Sasaki H (2002) A multi-agent approach to power system restoration. IEEE Trans Power Syst 17:457-462. https://doi.org/10. 1109/TPWRS.2002.1007918

13. Solanki JM, Khushalani S, Schulz NN (2007) A multi-agent solution to distribution systems restoration. IEEE Trans Power Syst 22: 1026-1034. https://doi.org/10.1109/TPWRS.2007.901280

14. Khamphanchai W, Pisanupoj S, Ongsakul W, Pipattanasomporn M (2011) A multi-agent based power system restoration approach in distributed smart grid. Util Exhib Power Energy Syst Issues Prospect Asia (ICUE), 2011 Int Conf. https://doi.org/10.1109/ ICUEPES.2011.6497754

15. Solanki JM, Schulz NN, Gao W (2005) Reconfiguration for restoration of power systems using a multi-agent system. Power Symp 2005 Proc 37th Annu North Am. https://doi.org/10.1109/NAPS. 2005.1560571

16. Khamphanchai W, Pipattanasomporn M, Rahman S (2012) A multi-agent system for restoration of an electric power distribution network with local generation. IEEE Power Energy Soc Gen Meet. https://doi.org/10.1109/PESGM.2012.6345106

17. Raju L, Morais AA, Rathnakumar R et al (2017) Micro-grid Grid Outage Management using Multi Agent Systems. Energy Procedia 117:112-119. https://doi.org/10.1016/j.egypro.2017.05.113

18. Trigo P, Marques P (2008) The electricity market as a multi-agent system. 2008 5th Int Conf Eur Electr Mark EEM. https://doi.org/10. 1109/EEM.2008.4579092

19. Logenthiran T, Srinivasan D (2011) Multi-agent system for market based microgrid operation in smart grid environment. Dept. Elec. Comp. Sci. National University of Singapore

20. Kok K (2010) Multi-agent coordination in the electricity grid, from concept towards market introduction. Proc 9th Int Conf Auton Agents Multiagent Syst Ind track

21. Santos G, Pinto T, Morais H et al (2015) Multi-agent simulation of competitive electricity markets: Autonomous systems cooperation for European market modeling. Energy Convers Manag 99:387399. https://doi.org/10.1016/j.enconman.2015.04.042
22. Tolbert LM, Qi H, Peng FZ (2001) Scalable multi-agent system for real-time electric power management. 2001 Power Eng Soc Summer Meet Conf Proc (Cat No01CH37262) 3:1-4. https://doi. org/10.1109/PESS.2001.970327

23. Zheng G, Li N (2010) Multi-Agent Based Control System for Multi-Microgrids. 2010 Int Conf Comput Intell Softw Eng. https://doi.org/10.1109/CISE.2010.5676818

24. Wang H (2001) Multi-agent co-ordination for the secondary voltage control in power-system contingencies. IEE Proc - Gener Transm Distrib 148:61-66. https://doi.org/10.1049/ip-gtd:20010025

25. Xu Y, Liu W, Gong J (2011) Stable multi-agent-based load shedding algorithm for power systems. IEEE Trans Power Syst 26: 2006-2014. https://doi.org/10.1109/TPWRS.2011.2120631

26. Eddy YSF, Gooi HB, Chen SX (2015) Multi-agent system for distributed management of microgrids. IEEE Trans Power Syst 30:24 34. https://doi.org/10.1109/TPWRS.2014.2322622

27. Abedini R, Pinto T, Morais H, Vale Z (2013) Multi-agent approach for power system in a smart grid protection context. 2013 IEEE Grenoble Conf PowerTech, POWERTECH 2013. https://doi.org/ 10.1109/PTC.2013.6652158

28. Wan H, Wong KP, Chung CY (2008) Multi-agent application in protection coordination of power system with distributed generations. IEEE Power Energy Soc 2008 Gen Meet Convers Deliv Electr Energy 21st Century, PES. https://doi.org/10.1109/PES. 2008.4596261

29. Do Nascimento LL, Rolim JG (2013) Multi-agent system for adaptive protection in microgrids. 2013 IEEE PES Conf Innov Smart Grid Technol ISGT LA 2013. https://doi.org/10.1109/ISGT-LA. 2013.6554435

30. Yong L, Tao S (2007) Economic dispatch of power system incorporating wind power plant. 2007 Int Power Eng Conf (IPEC 2007) 159-162. https://ieeexplore.ieee.org/document/4510019. Accessed 5 Jan 2018

31. Nagata T, Ohno M, Sasaki H, Fujita H (2002) A multi-agent approach to unit commitment. Electr Eng Japan 141:41-47. https:// doi.org/10.1002/eej.10057

32. Li N, Singhal NG, Hedman KW (2017) An enhanced securityconstrained unit commitment model with reserve response set policies. Proc. 50th Hawaii Int Conf Syst Sci. https://doi.org/10.24251/ hicss.2017.371

33. Kantamneni A, Brown LE, Parker G, Weaver WW (2015) Survey of multi-agent systems for microgrid control. Eng Appl Artif Intell 45:192-203. https://doi.org/10.1016/j.engappai.2015.07.005

34. Dimeas AL, Hatziargyriou ND (2004) A multiagent system for microgrids. IEEE Power Eng Soc Gen Meet 2:55-58. https://doi. org/10.1109/PES.2004.1372752

35. Wang Z, Yang R, Wang L (2011) Intelligent multi-agent control for integrated building and micro-grid systems. IEEE PES Innov Smart Grid Technol Conf Eur ISGT Eur. https://doi.org/10.1109/ISGT. 2011.5759134

36. Lü P, Zhao J, Yao J, Yang S (2017) A decentralized approach for frequency control and economic dispatch in smart grids. IEEE J Emerg Sel Top Circuits Syst 7:447-458. https://doi.org/10.1109/ JETCAS.2017.2708900

37. Nygard KE, Ghosn SB, Mcculloch R, et al (2012) Decision support independence in a smart grid. ENERGY 2012 Second Int Conf Smart Grids, Green Commun IT Energy-aware Technol Decis

38. Silvestre D, Hespanha JP, Silvestre C (2018) Broadcast and gossip stochastic average consensus algorithms in directed topologies. IEEE Trans Control Netw Syst. https://doi.org/10.1109/TCNS. 2018.2839341

39. Raffaelli G, Marsili M (2005) Statistical mechanics model for the emergence of consensus. Phys Rev E Stat Nonlinear Soft Matter Phys 72. https://doi.org/10.1103/PhysRevE.72.016114 
40. Gu D, Wang Z (2009) Leader-follower flocking: Algorithms and experiments. IEEE Trans Control Syst Technol 17:1211-1219. https://doi.org/10.1109/TCST.2008.2009461

41. Briñón-Arranz L, Schenato L (2013) Consensus-based sourceseeking with a circular formation of agents. In: Eur. Control Conf. pp 2831-2836. https://doi.org/10.23919/ECC.2013.6669782

42. Zhang X, Xu H, Yu T et al (2016) Robust collaborative consensus algorithm for decentralized economic dispatch with a practical communication network. Electr Power Syst Res 140:597-610. https:// doi.org/10.1016/j.epsr.2016.05.014

43. Hug G, Kar S, Wu C (2015) Consensus+Innovations Approach for Distributed Multiagent Coordination in a Microgrid. IEEE Trans Smart Grid 6:1893-1903. https://doi.org/10.1109/TSG.2015. 2409053

44. Lü P, Zhao J, Yao J, Yang S (2017) A decentralized approach for frequency control and economic dispatch in smart grids. IEEE J Emerg Sel Top Circuits Syst PP:1-12

45. Pipattanasomporn M, Feroze H, Rahman S (2009) Multi-agent systems in a distributed smart grid: Design and implementation. IEEE/PES Power Syst Conf Expo 2009 PSCE '09 1-8. https:// doi.org/10.1109/PSCE.2009.4840087

46. Kravari K, Bassiliades N (2015) A survey of agent platforms. JASSS. https://doi.org/10.18564/jasss.2661

47. Singh A, Juneja D, Sharma AK (2011) Agent Development Toolkits. Int J Adv Technol 2:158-164

48. Shoham Y (1991) AGENT0: A simple agent language and its interpreter. In: Proc. AAAI-91. pp 704-709

49. Thomas SR (1994) The PLACA agent programming language. In: Intell. Agents. pp 355-370

50. Bellifemine F, Caire G, Greenwood D (2007) Developing multiagent systems with JADE. John Wiley Sons Ltd. https://doi.org/10. 1002/9780470058411

51. Gutknecht O, Ferber J (2001) The MadKit agent platform architecture. In: Infrastruct. Agents, Multi-Agent Syst. Scalable MultiAgent Syst. SE - 5. pp 48-55

52. Guo Q, Han J, Yoon M, Jang G (2012) A study of economic dispatch with emission constraint in smart grid including wind turbines and electric vehicles. 2012 IEEE Veh Power Propuls Conf VPPC 2012 1002-1005. https://doi.org/10.1109/VPPC.2012.6422753

53. Nair AS, Ranganathan P, Salehfar H, Kaabouch N (2017) Uncertainty quantification of wind penetration and integration into smart grid: a survey. North Am. Power Symp. https://doi.org/10. 1109/NAPS.2017.8107196

54. Dominguez-Garcia AD, Cady ST, Hadjicostis CN (2012) Decentralized optimal dispatch of distributed energy resources. In: Proc. IEEE Conf. Decis. Control. pp 3688-3693. https://doi. org/10.1109/CDC.2012.6426665

55. Rana R, Bhattacharjee S, Mishra S A consensus based solution to the classic economic dispatch problem on a multi-agent system framework. In: 2018 IEEMA Eng. Infin. Conf. IEEE, pp 1-6. https://doi.org/10.1109/ETECHNXT.2018.8385372

56. Yu W, Li C, Yu X et al (2018) Economic power dispatch in smart grids: a framework for distributed optimization and consensus dynamics. Sci China Inf Sci 61:1-16. https://doi.org/10.1007/s11432016-9114-y

57. Zhang Z, Chow MY (2011) Incremental cost consensus algorithm in a smart grid environment. IEEE Power Energy Soc Gen Meet. https://doi.org/10.1109/PES.2011.6039422

58. Zhang Z, Chow M (2012) Convergence Analysis of the Incremental Cost Consensus Algorithm Under Different Communication Network Topologies in a Smart Grid. IEEE Trans Power Syst 27: 1761-1768

59. Zhang Z, Ying X, Chow MY (2011) Decentralizing the economic dispatch problem using a two-level incremental cost consensus algorithm in a smart grid environment. NAPS 2011 - 43rd North Am Power Symp. https://doi.org/10.1109/NAPS.2011.6025103
60. Yang S, Tan S, Xu J-X (2013) Consensus Based Approach for Economic Dispatch Problem in a Smart Grid. IEEE Trans Power Syst 28:4416-4426. https://doi.org/10.1109/TPWRS.2013. 2271640

61. Binetti G, Naso D, Turchiano B, et al (2014) Consensus-based approach for the economic dispatch problem. In: Proc. IFAC World Congr. IFAC, pp 3140-3145. https://doi.org/10.3182/ 20140824-6-ZA-1003.01786

62. Cherukuri A, Cortés J (2014) Distributed coordination for economic dispatch under varying loads and generator commitment. Fiftysecond Annu Allert Conf. https://doi.org/10.1109/allerton.2014. 7028493

63. Luo K, Wu Q, Jacob Ø, Hejde NA (2015) Real-time distributed economic dispatch for distributed generation based on multi-agent system. 2015 Mod. Electr. Power Syst. https://doi.org/10.1109/ MEPS.2015.7477174

64. Vergara PP, Shaker HR, Norregaard B, da Silva LCP (2017) Distributed consensus-based economic dispatch considering grid operation. 2017 IEEE Power Energy Soc Gen Meet. https://doi. org/10.1109/PESGM.2017.8273864

65. Guo F, Wen C, Mao J, Song YD (2016) Distributed Economic Dispatch for Smart Grids with Random Wind Power. IEEE Trans Smart Grid 7:1572-1583. https://doi.org/10.1109/TSG.2015.2434831

66. Cao J, Yu M, Tung LJ (2014) Consensus-based distributed control for economic dispatch problem with comprehensive constraints in a smart grid. EAI Endorsed Trans Energy Web 1:1-9. https://oi.org/ 10.4108/ew.1.2.e3

67. Yang Z, Xiang J, Li Y (2016) Distributed virtual incremental cost consensus algorithm for economic dispatch in a microgrid. IEEE Int Conf Control Autom ICCA. https://doi.org/10.1109/ICCA.2016. 7505307

68. Li Y-S, Zhang H-G, Huang B-N, Teng F (2016) Distributed optimal economic dispatch based on multi-agent system framework in combined heat and power systems. Appl Sci. https://doi.org/10.3390/ app6100308

69. Yang Z, Xiang J, Li Y (2017) Distributed consensus based supplydemand balance algorithm for economic dispatch problem in a smart grid with switching graph. IEEE Trans Ind Electron 64: 1600-1610. https://doi.org/10.1109/TIE.2016.2615037

70. Xing H, Mou Y, Fu M, Lin Z (2014) Consensus based bisection approach for economic power dispatch. Proc IEEE Conf Decis Control 2015-Febru. https://doi.org/10.1109/CDC.2014.7039979

71. Binetti G, Davoudi A, Naso D, et al (2014) A distributed auctionbased algorithm for the nonconvex economic dispatch problem. In: IEEE Trans. Ind. Informatics 10:1124-1132. https://doi.org/10. 1109/TII.2013.2287807

72. Binetti G, Davoudi A, Lewis FL et al (2014) Distributed consensusbased economic dispatch with transmission losses. IEEE Trans Power Syst 29:1711-1720. https://doi.org/10.1109/TPWRS.2014. 2299436

73. Zhabelova G, Vyatkin V, Zhang Z, Chow MY (2013) Agent-based distributed consensus algorithm for decentralized economic dispatch in Smart Grid. In: IECON Proc. (Industrial Electron. Conf. Vienna, Austria. https://doi.org/10.1109/IECON.2013.6699433

74. Wu C, Li H, Wu L, Wu Z (2015) A Multi-Agent Particle Swarm Optimization for Power System Economic Load Dispatch. J Comput Commun 3:83-89

75. Kumar R, Sharma D, Sadu A (2011) A hybrid multi-agent based particle swarm optimization algorithm for economic power dispatch. Int J Electr Power Energy Syst 33:115-123. https://doi.org/ 10.1016/j.ijepes.2010.06.021

76. Loia V, Vaccaro A (2014) Decentralized economic dispatch in smart grids by self-organizing dynamic agents. IEEE Trans Syst Man, Cybern Syst 44:397-408. https://doi.org/10.1109/TSMC.2013. 2258909 
77. Zhao C, He J, Cheng P, Chen J (2017) Analysis of ConsensusBased Distributed Economic Dispatch Under Stealthy Attacks. IEEE Trans Ind Electron 64:5107-5117

78. Li C, Yu X, Yu W et al (2016) Distributed event-triggered scheme for economic dispatch in smart grids. IEEE Trans Ind Informatics 12:1775-1785. https://doi.org/10.1109/TII.2015.2479558

79. Zhang Y, Rahbari-Asr N, Chow MY (2016) A robust distributed system incremental cost estimation algorithm for smart grid economic dispatch with communications information losses. J Netw Comput Appl 59:315-324. https://doi.org/10.1016/j.jnca.2015.05.014

80. Wen G, Yu X, Liu ZW, Yu W (2017) Adaptive Consensus-Based Robust Strategy for Economic Dispatch of Smart Grids Subject to Communication Uncertainties. IEEE Trans Ind Informatics 14: 2484-2496. https://doi.org/10.1109/TII.2017.2772088

81. Hamdi M, Chaoui M, Idoumghar L, Kachouri A (2018) Coordinated consensus for smart grid economic environmental power dispatch with dynamic communication network. IET Gener Transm Distrib 12:2603-2613. https://doi.org/10.1049/ietgtd.2017.1197

82. Yang T, Lu J, Wu D et al (2017) A Distributed Algorithm for Economic Dispatch over Time-Varying Directed Networks with Delays. IEEE Trans Ind Electron 64:5095-5106. https://doi.org/ 10.1109/TIE.2016.2617832

83. Wang R, Li Q, Zhang B, Wang L (2018) Distributed Consensus Based Algorithm for Economic Dispatch in a Microgrid. IEEE Trans Smart Grid 0:1-11. https://doi.org/10.1109/TSG.2018.2833108

84. Tang Z, Hill DJ, Liu T (2018) A Novel Consensus-Based Economic Dispatch for Microgrids. IEEE Trans Smart Grid 9:3920-3922. https://doi.org/10.1109/TSG.2018.2835657

85. He X, Yu J, Huang T, Li C (2018) Distributed power management for dynamic economic dispatch in the multimicrogrids environment. IEEE Trans Control Syst Technol 1-8. https://doi.org/10. 1109/TCST.2018.2816902

86. Lin P, Jin C, Xiao J et al (2018) A Distributed Control Architecture for Global System Economic Operation in Autonomous Hybrid AC/DC Microgrids. IEEE Trans Smart Grid 3053:1-13. https:// doi.org/10.1109/TSG.2018.2805839

87. Hajjar S, Bratcu AI, Hably A (2015) A day-ahead centralized unit commitment algorithm for a multi-agent smart grid. In: Fed Conf Comput Sci Inf Syst. pp 265-271. https://doi.org/10.15439/ $2015 f 227$
88. Yu J, Zhou J, Hua B, Liao R (2005) Optimal Short-Term Generation Scheduling with Multi-Agent System under a Deregulated Power. Int J Comput Cogn 3:61-65

89. Zhang $X$, Xie J, Zhu Z et al (2016) Smart grid cost-emission unit commitment via Co-evolutionary agents. Energies 9:1-13. https:// doi.org/10.3390/en9100834

90. Kaegi E, Cherkaoui R, Germond A (2008) Decentralized unit commitment and dispatch for the distribution systems using intelligent agents approach. In: PSCC 2008

91. Sharma D, Srinivasan D, Trivedi A (2011) Multi-agent approach for profit based unit commitment. In: 2011 IEEE Congr. Evol. Comput. CEC 2011. https://doi.org/10.1109/CEC.2011.5949932

92. Padhy NP (2004) Unit Commitment-A Bibliographical Survey. IEEE Trans Power Syst 19:1196-1205. https://doi.org/10.1109/ TPWRS.2003.821611

93. Carrión M, Arroyo JM (2006) A computationally efficient mixedinteger linear formulation for the thermal unit commitment problem. IEEE Trans Power Syst 21:1371-1378. https://doi.org/10. 1109/TPWRS.2006.876672

94. Lopez CJ, Ano O, Ojeda Esteybar D (2018) Stochastic Unit Commitment \&amp; Optimal Allocation of Reserves: A Hybrid Decomposition Approach. IEEE Trans Power Syst 8950:1-11. https://doi.org/10.1109/TPWRS.2018.2817639

95. Wang Y, Shahidehpour M, Lai LL et al (2018) ResilienceConstrained Hourly Unit Commitment in Electricity Grids. IEEE Trans Power Syst 8950:1-10. https://doi.org/10.1109/TPWRS. 2018.2817929

96. Xiong P, Singh C (2018) A Distributional Interpretation of Uncertainty Sets in Unit Commitment under Uncertain Wind Power. IEEE Trans Sustain Energy 3029:1-9. https://doi.org/10. 1109/TSTE.2018.2828421

97. Abedi S, He M, Member S, et al (2018) Congestion risk-aware unit commitment with significant wind power generation. IEEE Trans Power Syst 33:6861-6869. https://doi.org/10.1109/TPWRS.2018. 2831677

98. Nagata T, Ohono M, Kubokawa J et al (2002) A multi-agent approach to unit commitment problems. Proc IEEE Power Eng Soc Transm Distrib Conf 1:64-69. https://doi.org/10.1109/PESW.2002.984955

99. Yu J, Zhou J, Wu W, et al (2004) Solution of the profit-based unit commitment problem by using multi-agent system. In: WCICA 2004. Fifth World Congr. Intell. Control Autom. https://doi.org/ 10.1109/WCICA.2004.1343686 